\title{
Diagnostic accuracy of single-source dual-energy computed tomography and ultrasonography for detection of lateral cervical lymph node metastases of papillary thyroid carcinoma
}

\author{
Lin $\mathrm{Li}^{1}$, Sai-Nan Cheng ${ }^{1}$, Yan-Feng Zhao ${ }^{1 \#}$, Xiao-Yi Wang ${ }^{1}$, De-Hong Luo ${ }^{1}$, Yong Wang ${ }^{2 \#}$ \\ ${ }^{1}$ Department of Diagnostic Radiology, ${ }^{2}$ Department of Ultrasonography, National Cancer Center/National Clinical Research Center for Cancer/ \\ Cancer Hospital, Chinese Academy of Medical Sciences and Peking Union Medical College, Beijing 100021, China \\ Contributions: (I) Conception and design: YF Zhao, L Li; (II) Administrative support: DH Luo, Y Wang; (III) Provision of study materials or patients: \\ Y Wang, XY Wang, SN Cheng; (IV) Collection and assembly of data: L Li, SN Cheng, XY Wang; (V) Data analysis and interpretation: YF Zhao; \\ (VI) Manuscript writing: All authors; (VII) Final approval of manuscript: All authors. \\ "These authors contributed equally to this work. \\ Correspondence to: Yan-Feng Zhao. Department of Diagnostic Radiology, National Cancer Center/National Clinical Research Center for Cancer/ \\ Cancer Hospital, Chinese Academy of Medical Sciences and Peking Union Medical College, No. 17, Panjiayuannanli, Chaoyang District, Beijing \\ 100021, China. Email: zyf24@sina.com; Yong Wang. Department of Ultrasonography, National Cancer Center/National Clinical Research Center \\ for Cancer/Cancer Hospital, Chinese Academy of Medical Sciences and Peking Union Medical College, No. 17, Panjiayuannanli, Chaoyang District, \\ Beijing 100021, China. Email: drwangyong77@163.com.
}

Background: Dual-energy computed tomography (DECT) imaging can generate iodine-based material decomposition (MD) images and spectral HU curve. This study aimed to investigate the diagnostic accuracy of single-source dual-energy CT (DECT) and ultrasonography (US) for detecting lateral cervical lymph node metastases of papillary thyroid carcinoma (PTC).

Methods: Thirty patients with PTC were enrolled in the study and underwent DECT and US examination before thyroidectomy and cervical lymph node dissection. The spectral parameters included iodine concentration (IC), normalized iodine concentration (NIC) and slope $\left(\lambda_{\mathrm{HU}}\right)$ of lymph nodes. The CT morphological parameters included maximal short diameter, shape, margin, calcification and cystic degeneration of lymph nodes. The US morphological parameters included maximal short diameter, calcification and cystic degeneration of lymph nodes. The diagnostic value of every single spectral parameter, combined gemstone spectral image (GSI) parameters, CT morphological parameters and US morphological parameters between metastatic and non-metastatic lymph nodes were statistically compared. Receiver operating characteristic (ROC) curves, sensitivity, and specificity were used to determine the diagnostic value.

Results: Ninety-nine lymph nodes from thirty patients were pathologically confirmed. Among them, $70(70.7 \%)$ were metastatic. For single GSI parameters, ROC analysis showed that the area under the curve (AUC) for IC was the highest (AUC =0.937) but the difference was not statistically significant when compared with NIC or slope $\left(\lambda_{\mathrm{HU}}\right)(\mathrm{P}>0.05)$. The optimal diagnostic threshold for IC was $2.56 \mathrm{mg} / \mathrm{mL}$, with a sensitivity, specificity and accuracy of $87.1 \%, 93.1 \%$, and $88.9 \%$, respectively. The AUC for combined GSI parameter (AUC $=0.942$ ) was higher compared with the US morphological parameters (AUC $=0.771$, $\mathrm{P}<0.001$ ), with a sensitivity, specificity, and accuracy of $92.9 \%, 86.2 \%$, and $90.9 \%$, respectively. However AUC did not differ significantly among combined GSI parameters, combined CT morphological parameters and a single application for spectral CT parameters IC (P>0.05).

Conclusions: Combined GSI parameters showed better diagnostic accuracy in lateral cervical lymph node metastasis of PTC compared with that of combined US morphological parameters. IC alone showed excellent diagnostic stability and could be performed easily.

Keywords: Papillary thyroid carcinoma (PTC); lymph node; metastasis; dual-energy computed tomography (DECT); ultrasonography (US) 
Submitted Sep 10, 2019. Accepted for publication Dec 10, 2019.

doi: $10.21037 /$ jtd.2019.12.45

View this article at: http://dx.doi.org/10.21037/jtd.2019.12.45

\section{Introduction}

Papillary thyroid carcinoma (PTC) is a common malignant tumor of the neck. The lymph node metastasis rate of thyroid carcinoma was reported as from $35 \%$ to $80 \%(1-5)$. Although cervical lymph node metastasis does not affect the overall and disease-specific survival, it increases the risk of local recurrence. It was reported that regional lymph node metastasis accounted for $31 \%$ of local recurrences $(6,7)$. Therefore, accurate preoperative diagnosis of lymph node metastasis and consequently, neck dissection may reduce the recurrence rate. American Thyroid Association (ATA) guidelines (8) recommend that prophylactic centralcompartment neck dissection (ipsilateral or bilateral) should be considered in PTC patients, especially for advanced primary tumors (T3 or T4). However prophylactic lateral compartment neck dissection is not mentioned in the guidelines. Neck dissection may lead to severe postoperative complications, such as recurrent laryngeal nerve palsy, hematoma, chyle leakage, and spinal accessory nerve dysfunction (9). Therefore, the indications for neck dissection should be carefully investigated. Accurate preoperative diagnosis of the lymph node metastasis is important in determining the need for lateral compartment neck dissection in PTC patients.

Currently, the method of preoperative diagnosis of lymph node metastasis mainly includes ultrasonography (US) and computed tomography (CT), but both have limited specificity and even when these modalities are combined, there are still some limitations (10-14). Dualenergy computed tomography (DECT) imaging can reconstruct material decomposition (MD) images and a set of monochromatic images with photon energy ranging from 40 to $140 \mathrm{keV}$, to generate spectral HU curve. The added MD images and spectral HU curve can help diagnose the cervical lymph node metastasis of PTC (15). The aim of this study was to investigate the diagnostic accuracy of singlesource dual-energy CT (DECT) and US for detecting lateral cervical lymph node metastasis of PTC.

\section{Methods}

\section{Patient selection}

The study was approved by the Institutional Ethics Review
Board (National Cancer Center/National Clinical Research Center for Cancer/Cancer Hospital, Chinese Academy of Medical Sciences and Peking Union Medical College NCC2016 NZ-03) and all patients were provided with written informed consent. Our inclusion criteria were as follows: (I) age over 18 years; (II) thyroid cancer confirmed by postoperative pathology; (III) no other history of cervical tumors except the thyroid tumor; and (IV) no history of radiation treatment in the neck. The exclusion criteria were as follows: (I) history of surgical treatment or postoperative pathology confirmed to be benign lesions; (II) pathology confirmed to be malignant tumor other than PTC; (III) lymph nodes in CT images measured less than $5 \mathrm{~mm}$ in maximal short diameter and (IV) patients didn't receive cervical lymph node dissection.

\section{CT scanning protocol}

All CT scans were performed on a Discovery CT750 HD Scanner (GE Healthcare) using GSI mode. Patients were in a supine position, with the neck stretching as far as possible. The scanning range was from the skull base to the upper margin of the aortic arch. The scanning was performed using the following parameters: fast tube voltage switching $(0.5 \mathrm{~ms})$ between 80 and $140 \mathrm{kVp}$, tube current $=260 \mathrm{~mA}$, pitch $=0.984: 1$, rotation time $=0.7 \mathrm{~s}$, and reconstruction slice thickness and interval $=5 \mathrm{~mm}$. All patients were injected with $90 \mathrm{~mL}$ of the non-ionic contrast agent by using a highpressure injector at a flow rate of $3 \mathrm{~mL} / \mathrm{s}$. The scan was started $45 \mathrm{~s}$ after the injection of the non-ionic contrast agent.

All images with data file were transferred to an advantage workstation (AW4.6. GE Healthcare) to generate 40, 70 and $100 \mathrm{keV}$ images, iodine-based MD images and spectral HU curve which were analyzed by a single radiologist with 17 years of experience in head and neck cancer diagnosis who was blinded to the pathological results. Images measurements and analysis were performed by using the GSI viewer, an image analysis software. The CT morphological parameters included: maximal short diameter of the lymph node on the cross-sectional plane; shape and margin; small calcification and cystic degeneration (11,16-20).

The CT GSI parameters were measured using $70 \mathrm{keV}$ images by another radiologist with more than 5 years of experience in head and neck cancer diagnosis. Region- 
of-interests (ROIs) were placed on the lymph nodes with maximal short diameter $\geq 5 \mathrm{~mm}$ while avoiding necrosis and calcification regions on the image slice containing the highest intensity. The parameters were measured three times and averaged. The GSI parameters (21-23) included the iodine concentration (IC) of ROIs, CT attenuation value (HU) of ROIs, IC of the common carotid artery on the same image slice, normalized iodine concentration (NIC) and slope $\left(\lambda_{\mathrm{HU}}\right)$. The formula of NIC and $\lambda_{\mathrm{HU}}$ were as follows: NIC $=$ $\mathrm{IC}_{\text {lesion }} / \mathrm{IC}_{\text {common carotid artery }} \lambda_{\mathrm{HU}}=\left[\mathrm{CT}\right.$ value ${ }_{(40 \mathrm{keV})}-\mathrm{CT}$ value $\left.{ }_{(100 \mathrm{keV})}\right] /(100-40)$. "CT value ${ }_{(40 \mathrm{keV})}$ " and "CT value ${ }_{(100 \mathrm{keV})}$ " are the CT attenuation measurements at 40 and $100 \mathrm{keV}$, respectively.

\section{US}

All preoperative US examinations were performed by one radiologist with 19 years of experience in thyroid imaging using a US equipment (GE-Logiq9, GE Healthcare, WI) with a $10-14 \mathrm{MHz}$ transducer. The US morphological parameters included maximal short diameter, calcification and cystic degeneration of lymph nodes $(1,11,24,25)$.

\section{Surgical patbology analysis and grouping}

Lymph nodes were divided into 7 regions bilaterally (a total of 14 regions) according to Som's criteria (26) in radiological, surgical and pathological assessment. Based on US, CT results and frozen section pathologic analysis during surgery, different surgical procedures were chosen for thyroid diseases and lymph nodes. Prophylactic central compartment neck dissections were performed in patients when there was suspicion of PTC. Ipsilateral or bilateral lateral compartment dissections were performed when there was suspicion of metastatic lymph nodes. When suspected metastasis lymph node shown in an area, the entire regional lymph nodes were resected. The lymph nodes were marked by surgeons according to the lymph node region and were sent for pathologic examination. Surgical specimens were stained and diagnosed by experienced pathologists. CT images were compared with the pathological analysis according to the lymph node classification. All lymph nodes were divided into metastasis group and non-metastasis group.

\section{Statistical analysis}

Continuous variables were present as the mean \pm standard deviation (SD). Categorical variables were presented as rate. We used either the $\chi^{2}$ (Pearson method and McNemar method) test or the Fisher exact test for categorical variables and the independent $t$ test for continuous variables to compare the differences between metastasis group and non-metastasis group. The receiver operating characteristic (ROC) curve analyses were used to determine the discrimination ability of various image parameters for lymph node metastasis, using postoperative pathological results as the gold standard. The best cutoff values were calculated by the Youden index, and the sensitivity, specificity, and accuracy of these cutoff values were also calculated. All statistical analyses were performed using SPSS 20.0 software (SPSS, Chicago, Illinois, USA). Statistical figures were drawn using GraphPad Prism 7.0 (GraphPad Software, San Diego, CA, USA). The area under the ROC curves (AUC) was compared using MedCalc 18.2.1 (MedCalc Software, Ostend, Belgium). $\mathrm{P}$ values $<0.05$ were considered to indicate statistical significance.

\section{Results}

\section{Patients and lymph nodes}

From January 2017 to December 2017, 108 consecutive patients underwent DECT and US on their first visit to our hospital due to the presence of a thyroid mass. According to the exclusion criteria, 78 patients were excluded: postoperative pathology confirmed nodular goiter, adenoma, thyroiditis and other benign lesions (22 cases); pathology confirmed lymphoma (2 cases), medullary carcinoma (2 cases); lymph nodes in CT images measured less than $5 \mathrm{~mm}$ in maximal short diameter and patients didn't receive cervical lymph node dissection (52 cases). Finally, 30 patients were included in the study. The mean age of the 30 patients [ 13 men $(43.3 \%)$ and 17 women $(56.7 \%)$ ] was $41.6 \pm 14.8$ years (range, $18-66$ years). A total of ninetynine lymph nodes from thirty patients were pathologically confirmed. Among them, 70 (70.7\%) were metastatic, 29 were non-metastatic. The distribution of lymph nodes is shown in Table 1.

None of the region I lymph nodes were dissected in any of all the patients, and thus was not included.

\section{Morphological features of lymph nodes}

The CT (maximal short diameter, shape, margin, calcification and cystic degeneration) and US (maximal 
short diameter, calcification, and cystic degeneration) morphological features of lymph nodes in the metastatic and non-metastatic groups are shown in Table 2. Table 2 also shows the sensitivity, specificity, and accuracy of each feature in diagnosing lymph node metastasis, irregular shape, unclear margin, calcification, and cystic degeneration were associated with malignant lymph nodes (Figures 1-3).

\section{GSI parameters of lymph nodes}

The GSI parameters (IC, NIC, and $\lambda_{\mathrm{HU}}$ ), the optimal cut-

Table 1 Distribution of metastatic and non-metastatic lymph nodes

\begin{tabular}{lccccc}
\hline Group & II & III & IV & V & Total \\
\hline Metastatic & 11 & 22 & 31 & 6 & 70 \\
Non-metastatic & 11 & 12 & 6 & 0 & 29 \\
Total & 22 & 34 & 37 & 6 & 99 \\
\hline
\end{tabular}

off value and the sensitivity, specificity and accuracy of each parameter in diagnosing lymph nodes metastasis are shown in Table 3.

IC, NIC, and $\lambda_{\mathrm{HU}}$ for the lymph node metastasis group were statistically higher than the non-metastasis group $(\mathrm{P}<0.001)$ (Figure 4, Table 3). Although the AUC of IC was the highest (0.937) among the three GSI parameters, there was no significant difference when compared with the other two parameters $(\mathrm{P}>0.05)$ (Figure 5).

\section{The diagnostic value of combined GSI, CT morphological and US morphological parameters}

All positive parameters were assigned to class 1 (or 0 ), including $\mathrm{IC}>2.56 \mathrm{mg} / \mathrm{mL}, \mathrm{NIC}>0.29, \lambda_{\mathrm{HU}}>2.69$, maximal short diameter $(\mathrm{CT})>0.8 \mathrm{~cm}$, irregular shape $(\mathrm{CT})$, unclear margin (CT), calcification (CT), cystic degeneration (CT), maximal short diameter (US) $>0.9 \mathrm{~cm}$, calcification (US), and cystic degeneration (US). The ROC curves

Table 2 CT and US morphological signs of metastatic and non-metastatic lymph nodes

\begin{tabular}{|c|c|c|c|c|c|c|c|c|c|c|}
\hline Technique & Parameter & Characteristic & $\begin{array}{c}\text { Metastatic } \\
\quad(\mathrm{n}=70)\end{array}$ & $\begin{array}{l}\text { Non-metastatic } \\
\quad(n=29)\end{array}$ & Statistic & $P$ value & Cutoff value & $\begin{array}{c}\text { Sensitivity } \\
(\%)\end{array}$ & $\begin{array}{c}\text { Specificity } \\
(\%)\end{array}$ & $\begin{array}{c}\text { Accuracy } \\
\text { (\%) }\end{array}$ \\
\hline \multirow[t]{7}{*}{ CT } & $\begin{array}{l}\text { Maximal short } \\
\text { diameter }\end{array}$ & Mean (cm) & 1.14 & 0.64 & 4.466 & 0.000 & $>0.80$ & 61.4 & 93.1 & 70.7 \\
\hline & & $\mathrm{SD}(\mathrm{cm})$ & 0.60 & 0.16 & & & & & & \\
\hline & Shape & Irregular (n) & 32 & 0 & 19.589 & 0.000 & Irregular & 45.7 & 100.0 & 61.6 \\
\hline & Margin & Unclear (n) & 26 & 0 & 14.608 & 0.000 & Unclear & 37.1 & 100.0 & 55.6 \\
\hline & & Clear (n) & 44 & 29 & & & & & & \\
\hline & Calcification & Yes (n) & 13 & 0 & 6.200 & 0.013 & Calcification & 18.6 & 100.0 & 42.4 \\
\hline & & No $(n)$ & 57 & 29 & & & & & & \\
\hline \multirow[t]{6}{*}{ US } & $\begin{array}{l}\text { Maximal short } \\
\text { diameter }\end{array}$ & Mean $(\mathrm{cm})$ & 1.54 & 0.92 & 2.857 & 0.005 & $>0.90$ & 68.6 & 82.8 & 72.7 \\
\hline & & $\mathrm{SD}(\mathrm{cm})$ & 1.05 & 0.80 & & & & & & \\
\hline & Calcification & Yes (n) & 34 & 8 & 3.697 & 0.055 & Calcification & 48.6 & 72.4 & 55.6 \\
\hline & & No $(n)$ & 36 & 21 & & & & & & \\
\hline & $\begin{array}{c}\text { Cystic } \\
\text { degeneration }\end{array}$ & Yes (n) & 13 & 0 & 6.200 & 0.013 & $\begin{array}{c}\text { Cystic } \\
\text { degeneration }\end{array}$ & 18.6 & 100.0 & 42.4 \\
\hline & & No $(n)$ & 57 & 29 & & & & & & \\
\hline
\end{tabular}

US, ultrasonography. 

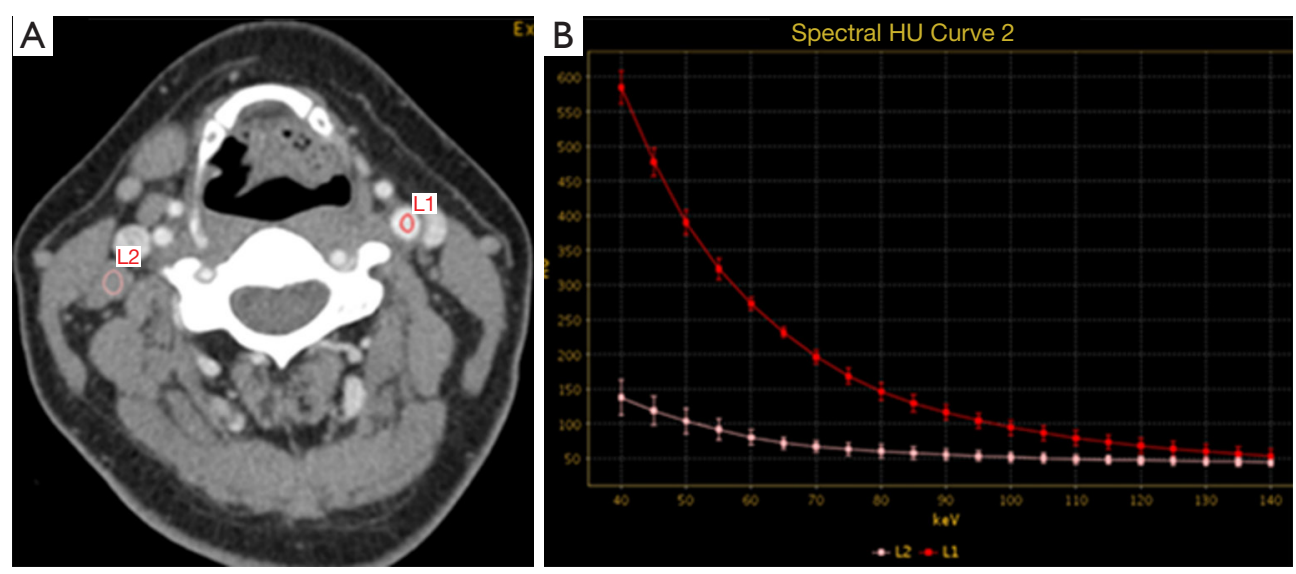

Figure 1 Papillary thyroid carcinoma without lateral cervical lymph node metastases. (A) Enhanced $70 \mathrm{keV}$ image at axial view shows an enlarged lymph node in right cervical region II, with the absent of significant enhancement. ROI L1 is put on the left common carotid artery. ROI L2 is put on the right cervical region II lymph node. (B) The spectral curve shows that the spectral curve of right cervical region II lymph node (L2 pink curve) is below that of the left common carotid artery (L1 red curve). ROI, region-of-interest.
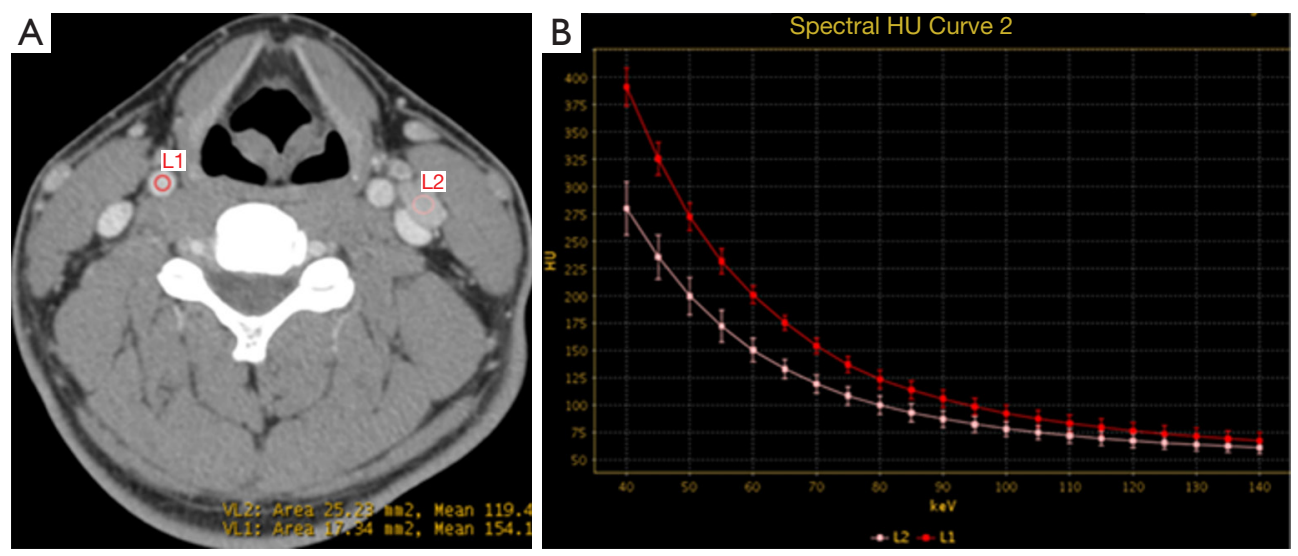

Figure 2 Papillary thyroid carcinoma with lateral cervical lymph node metastases. (A) Enhanced $70 \mathrm{keV}$ image at axial view shows an enlarged lymph node in left cervical region III, with significant enhancement. ROI L1 is put on the right common carotid artery. ROI L2 is put on the left cervical region III lymph node. (B) The spectral curve shows that the spectral curve of the left cervical region III lymph node (L2 pink curve) is close to that of the right common carotid artery (L1 red curve). ROI, region-of-interest.

of combined GSI parameters (IC, NIC, $\lambda_{\mathrm{HU}}$ ), combined CT morphological parameters (maximal short diameter, shape, margin, calcification, cystic degeneration) and combined US morphological parameters (maximal short diameter, calcification, and cystic degeneration) were drawn respectively (Figure 6). The optimal cut-off value, the sensitivity, specificity, and accuracy of each set of combined parameters in diagnosing lymph node metastasis are shown in Table 4. The combined GSI parameters (the presence of any 2 of the parameters) showed the highest diagnostic value $(\mathrm{AUC}=0.942$, sensitivity $=92.9 \%$, specificity $=86.2 \%$, and accuracy $=90.9 \%$ ), with a significant difference when compared with the combined US morphological parameters (presence of any of the parameters, AUC $=0.771$, sensitivity $=78.6 \%$, specificity $=65.5 \%$, accuracy $=74.7 \%, \mathrm{P}<0.001$ ), There was no significant difference when compared with the combined CT morphological parameters (presence of any 3 of the parameters, $\mathrm{AUC}=0.879$, sensitivity $=78.6 \%$, specificity $=93.1 \%$, accuracy $=82.8 \%, \mathrm{P}>0.05)$. The diagnostic value of IC alone (AUC $=0.937$ ) was lower than that of the combined GSI parameters, but the difference was not statistically significant. The diagnostic value of IC 
alone was significantly higher than that of combined US morphological parameters $(\mathrm{z}=0.4017, \mathrm{P}<0.001)$.

\section{Discussion}

PTC is the most common malignant tumor of the thyroid gland with a high rate of lymph node metastasis. ATA guidelines (8) recommend that prophylactic centralcompartment neck dissection (ipsilateral or bilateral) should be considered in PTC patients. However there is no explicit

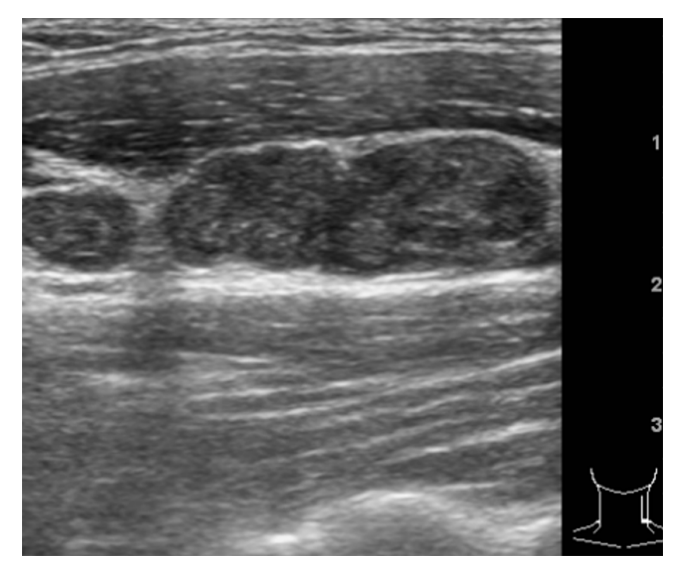

Figure 3 High frequency ultrasound image shows enlarged left cervical lymph nodes, with microcalcification. consensus regarding prophylactic lateral compartment neck dissection. For the lymph nodes that which were preoperatively diagnosed as non-metastatic lymph nodes, the dissection will not extend the disease-free survival time but may lead to severe postoperative complications (9). Therefore, preoperative lymph nodes assessment is critical to surgical planning and prognosis. Traditional methods of diagnosing lymph node metastasis include palpation, US, CT, PET-CT, and fine-needle aspiration. Palpation has been replaced by US, CT, and PET-CT (27-30) due to its limited sensitivity. According to previous studies, the sensitivity of using US and CT for diagnosing lateral lymph node metastasis are $64.0-74.3 \%$ and $68.6-78.2 \%$, the specificity of US and CT for lateral lymph node metastasis is $82-94.8 \%$ and $78-95 \%(3,11,17-20,31-33)$, respectively. The sensitivity and specificity of US is similar to that of CT scan in the lateral neck. Previous studies have shown that US had high specificity (82-94.8\%) but low sensitivity (64.0-74.3\%) for preoperative detection of cervical nodal metastasis in patients with PTC. In our study, the sensitivity of US and CT for lateral lymph node metastasis is $78.6 \%$ and $78.6 \%$, and specificities is $65.5 \%$ and $93.3 \%$, respectively, in agreement with previous studies. Even though some studies considered US to be superior to CT in diagnosing lymph node metastasis $(1,24,31-33)$. The role of CT in clinical practice cannot be neglected because US is

Table 3 The GSI parameters of lymph node metastasis and non-metastasis groups

\begin{tabular}{|c|c|c|c|c|c|c|c|c|}
\hline Parameter & Metastasis $(n=70)$ & Non-metastasis $(n=29)$ & $t^{a}$ & $\mathrm{P}^{\mathrm{a}}$ & Cutoff value & Sensitivity (\%) & Specificity (\%) & Accuracy (\%) \\
\hline $\mathrm{NIC}$ & $0.73 \pm 0.61$ & $0.28 \pm 0.16$ & 5.599 & $<0.001$ & $>0.29$ & 97.1 & 75.9 & 90.9 \\
\hline $\operatorname{Slope}\left(\lambda_{H U}\right)$ & $4.76 \pm 2.04$ & $2.21 \pm 2.93$ & 8.549 & $<0.001$ & $>2.69$ & 91.4 & 86.2 & 89.9 \\
\hline
\end{tabular}

a , stands for $t$-test between the metastasis group and the non-metastasis group. GSI, gemstone spectral image; IC, iodine concentration; NIC, normalized iodine concentration.
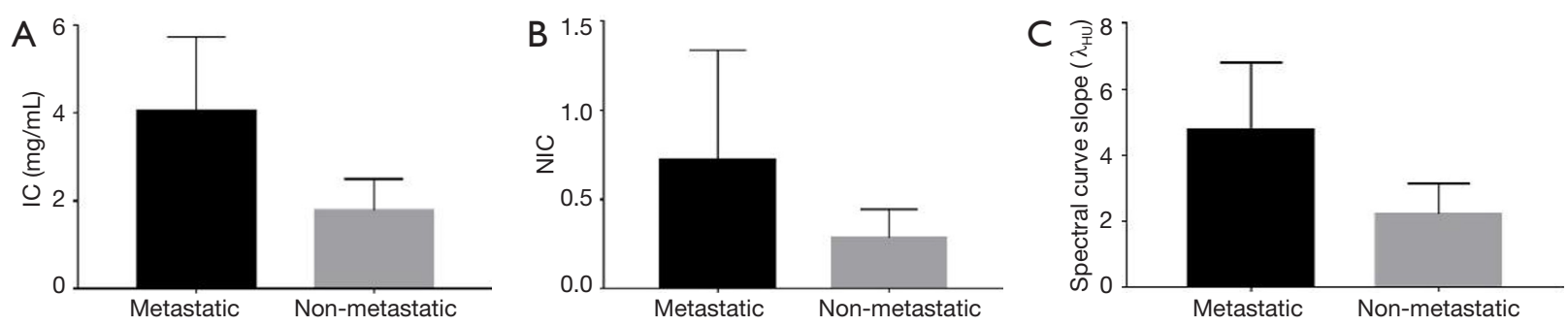

Figure 4 IC (A), NIC (B) and $\lambda_{\mathrm{HU}}(\mathrm{C})$ for lymph node metastasis and non-metastasis groups. IC, iodine concentration; NIC, normalized iodine concentration. 


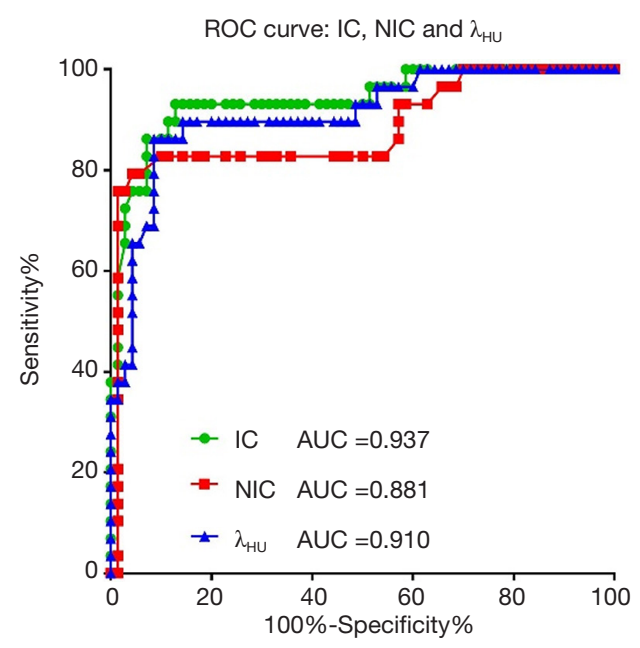

Figure 5 ROC curves of IC, NIC, and $\lambda_{\mathrm{HU}}$ in diagnosing lymph nodes metastasis. ROC, receiver operating characteristic; IC, iodine concentration; NIC, normalized iodine concentration.

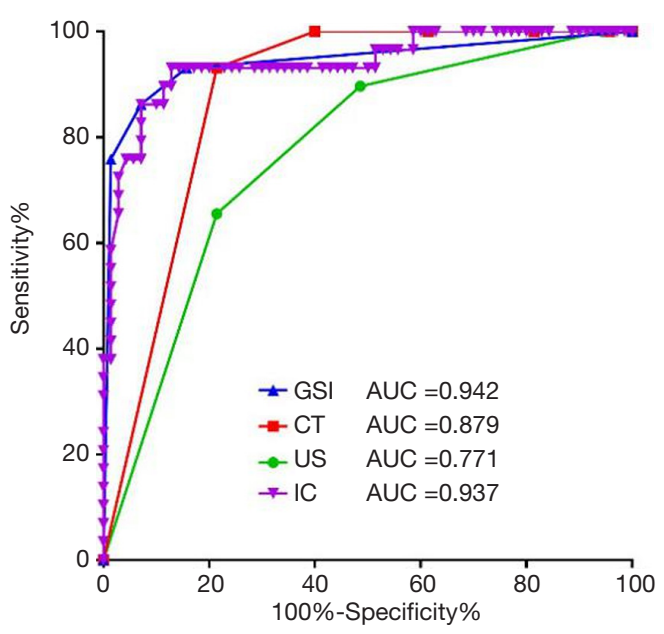

Figure 6 The ROC curves of combined GSI, combined CT morphological, combined US morphological parameters and single parameter IC. ROC, receiver operating characteristic; GSI, gemstone spectral image; CT, computed tomography; US, ultrasonography; IC, iodine concentration.

Table 4 The diagnostic value of combined GSI, combined CT morphological parameters, combined US morphological parameters and a single parameter IC

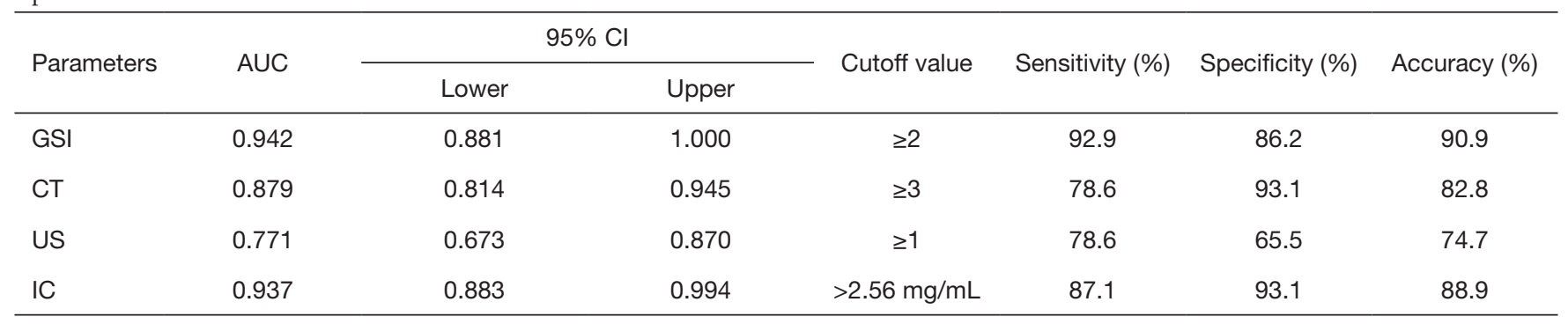

GSI = combined GSI parameter; CT = combined CT morphological parameters; US = combined US morphological parameters; IC = a single parameter IC. AUC, area under the curve; GSI, gemstone spectral image; CT, computed tomography; US, ultrasonography; IC, iodine concentration.

often dependent on operators $(1,18,34,35)$.

CT morphological parameters such as size, shape, margins, calcification and cystic degeneration have been widely used to diagnose lymph node metastasis of thyroid cancer $(18,34,35)$. Although the shape and margin features of lymph nodes contribute to the diagnosis of metastasis, the signs are significantly influenced by the observer's subjective assessment and have limited reliability. In our study, the sensitivity $(45.7 \%, 37.1 \%)$ and accuracy $(61.6 \%$, $55.6 \%)$ of shape and margin on conventional CT imaging were low, and the diagnostic value was limited. Calcification and cystic degeneration are easily detected by CT, with high specificity. However similar to previous studies $(3,11,19,20)$, we also found that the sensitivity $(18.6 \%, 37.1 \%)$ and accuracy $(42.4 \%, 55.6 \%)$ were very low, resulting in limited practical value.

The normal thyroid gland can uptake iodine. PTC and its metastases can also uptake iodine (36,37). Enhanced spectral CT imaging can reconstruct both iodine-based MD images and monochromatic spectral images. Iodinebased MD images can be used to estimate quantitatively IC. Monochromatic spectral images can be used to generate a spectral HU curve for calculating its slope. The added MD images and spectral HU curve can help differentiate benign and malignant lesions $(21-23,38)$, especially in diagnosing the cervical lymph node metastasis of PTC (15). In our 
study, we found that the IC, NIC and $\lambda_{\mathrm{HU}}$ of metastatic group was significantly higher than that in non-metastatic group, similar results also were reported in previous studies (15). Our results indicated that GSI parameters (IC, NIC, $\left.\lambda_{\mathrm{HU}}\right)$ were valuable in diagnosing lateral cervical lymph node metastasis in PTC patients. The diagnostic value of IC alone was significantly higher than that of the combined US morphological parameters (AUC: 0.937 vs. 0.771 , $\mathrm{P}<0.05)$. The combined GSI parameters showed higher diagnostic value compared with IC, but the difference was not statistically significant. This may partially be due to the high value of each parameter. Besides, NIC was calculated based on IC. Therefore, there was no significant difference between combined GSI parameters and IC in terms of the diagnostic value for cervical lymph node metastasis. Increased IC may be related to the increased tumor blood supply and consequently increased uptake of iodine (36,37,39-41).

The sensitivity of IC (cut-off value $2.56 \mathrm{mg} / \mathrm{mL}$ ) in the diagnosis of lymph node metastasis was $87.1 \%$. Nine lymph nodes gave a false negative result, 3 of which showed microcalcification while 3 showed cystic degeneration in CT images. The sensitivity of spectral CT imaging may improve to $95.7 \%$ if the 6 lymph nodes were diagnosed with metastases. IC had a high value for the diagnosis of lymph node metastasis and was less affected by the observer's subjective judgment. Combining CT morphological signs such as calcification and cystic degeneration with GSI parameters such as IC could further improve the diagnostic accuracy.

There are several limitations to this study. First, the sample size was small, ninety-nine lymph nodes from thirty patients were included in the study, which results in a certain level of homology, reducing the reliability of the results. Thus, their representativeness is limited. However, the value of this study is to explore the feasibility of this method, especially to use the multi-parameter objective index of spectral CT to replace the traditional US and CT methods with subjective judgment as the main diagnostic basis. Nonetheless, a small number of statistically significant differences were elucidated. These cases represent 30 patients, thus showing that the study method has certain representativeness and repeatability. We still need to further expand the sample size for analysis and comparison. Second, the prophylactic lateral compartment neck dissection is not mentioned in the guidelines. Lateral compartment neck dissection was not performed if there were negative findings in the imaging evaluations. Therefore, the number of metastatic lymph nodes was significantly higher than that of non-metastatic lymph nodes, which may lead to bias in the results and may potentially affect sensitivity and accuracy. Furthermore, all lymph nodes assessments were performed by one experienced radiologist, and we did not examine interobserver agreement. The study aimed to compare the diagnostic value of three methods: GSI parameters, CT morphology, and ultrasound morphology. Parameters of GSI is a new method for diagnosing lymph node metastases. It is an objective method. In contrast, CT morphology and ultrasound morphology are subjective diagnostic methods, but they are classic diagnostic methods.

\section{Conclusions}

Compared with qualitative assessment with US morphological parameters, GSI parameters showed a higher diagnostic value for detection of lateral cervical lymph node metastasis in PTC patients. IC alone showed excellent diagnostic stability and could be performed easily. Combining CT morphological signs such as calcification and cystic degeneration with spectral CT specific parameters such as IC could further improve the diagnostic value.

\section{Acknowledgments}

Funding: This work was supported by Beijing Hope Run Special Fund of Cancer Foundation of China (LC2016A13).

\section{Footnote}

Conflicts of Interest: The authors have no conflicts of interest to declare.

Ethical Statement: The authors are accountable for all aspects of the work in ensuring that questions related to the accuracy or integrity of any part of the work are appropriately investigated and resolved. This study was approved by the Institutional Ethics Review Board (National Cancer Center/National Clinical Research Center for Cancer/Cancer Hospital, Chinese Academy of Medical Sciences and Peking Union Medical College NCC2016 NZ-03). Each patient was consent informed.

\section{References}

1. Lesnik D, Cunnane ME, Zurakowski D, et al. Papillary 
thyroid carcinoma nodal surgery directed by a preoperative radiographic map utilizing CT scan and ultrasound in all primary and reoperative patients. Head Neck 2014;36:191-202.

2. Sherman SI. Thyroid carcinoma. Lancet 2003;361:501-11.

3. Kim E, Park JS, Son KR, et al. Preoperative diagnosis of cervical metastatic lymph nodes in papillary thyroid carcinoma: comparison of ultrasound, computed tomography, and combined ultrasound with computed tomography. Thyroid 2008;18:411-8.

4. Gonzalez HE, Cruz F, O'Brien A, et al. Impact of preoperative ultrasonographic staging of the neck in papillary thyroid carcinoma. Arch Otolaryngol Head Neck Surg 2007;133:1258-62.

5. Han Z, Lei Z, Li M, et al. Differential diagnosis value of the ultrasound gray scale ratio for papillary thyroid microcarcinomas and micronodular goiters. Quant Imaging Med Surg 2018;8:507-13.

6. Simon D, Goretzki PE, Witte J, et al. Incidence of regional recurrence guiding radicality in differentiated thyroid carcinoma. World J Surg 1996;20:860-6; discussion 866.

7. Hay ID, Thompson GB, Grant CS, et al. Papillary thyroid carcinoma managed at the Mayo Clinic during six decades (1940-1999): temporal trends in initial therapy and longterm outcome in 2444 consecutively treated patients. World J Surg 2002;26:879-85.

8. American Thyroid Association (ATA) Guidelines Taskforce on Thyroid Nodules and Differentiated Thyroid Cancer, Cooper DS, Doherty GM, et al. Revised American Thyroid Association management guidelines for patients with thyroid nodules and differentiated thyroid cancer. Thyroid 2009;19:1167-214.

9. Cheah WK, Arici C, Ituarte PH, et al. Complications of neck dissection for thyroid cancer. World J Surg 2002;26:1013-6.

10. Wang Y, Guan Q, Xiang J, et al. Clinicopathologic features and prognostic factors of diffuse sclerosing variant of papillary thyroid carcinoma: a population-based analysis. Transl Cancer Res 2018;7:695-705.

11. Lee DW, Ji YB, Sung ES, et al. Roles of ultrasonography and computed tomography in the surgical management of cervical lymph node metastases in papillary thyroid carcinoma. Eur J Surg Oncol 2013;39:191-6.

12. Yoo YH, Kim JA, Son EJ, et al. Sonographic findings predictive of central lymph node metastasis in patients with papillary thyroid carcinoma: influence of associated chronic lymphocytic thyroiditis on the diagnostic performance of sonography. J Ultrasound Med 2013;32:2145-51.
13. Jin LX, Moley JF. Surgery for lymph node metastases of medullary thyroid carcinoma: A review. Cancer 2016;122:358-66.

14. Liu C, Chen S, Yang Y, et al. The value of the computeraided diagnosis system for thyroid lesions based on computed tomography images. Quant Imaging Med Surg 2019;9:642-53.

15. Zhao Y, Li X, Li L, et al. Preliminary study on the diagnostic value of single-source dual-energy CT in diagnosing cervical lymph node metastasis of thyroid carcinoma. J Thorac Dis 2017;9:4758-66.

16. Mack MG, Rieger J, Baghi M, et al. Cervical lymph nodes. Eur J Radiol 2008;66:493-500.

17. Kim DW, Choo HJ, Lee YJ, et al. Sonographic features of cervical lymph nodes after thyroidectomy for papillary thyroid carcinoma. J Ultrasound Med 2013;32:1173-80.

18. Yoon JH, Kim JY, Moon HJ, et al. Contribution of computed tomography to ultrasound in predicting lateral lymph node metastasis in patients with papillary thyroid carcinoma. Ann Surg Oncol 2011;18:1734-41.

19. Choi YJ, Yun JS, Kook SH, et al. Clinical and imaging assessment of cervical lymph node metastasis in papillary thyroid carcinomas. World J Surg 2010;34:1494-9.

20. Wu CW, Dionigi G, Lee KW, et al. Calcifications in thyroid nodules identified on preoperative computed tomography: patterns and clinical significance. Surgery 2012;151:464-70.

21. De Cecco CN, Darnell A, Rengo M, et al. Dual-energy CT: oncologic applications. AJR Am J Roentgenol 2012;199:S98-105.

22. Liu X, Ouyang D, Li H, et al. Papillary thyroid cancer: dual-energy spectral CT quantitative parameters for preoperative diagnosis of metastasis to the cervical lymph nodes. Radiology 2015;275:167-76.

23. Lv P, Lin XZ, Li J, et al. Differentiation of small hepatic hemangioma from small hepatocellular carcinoma: recently introduced spectral CT method. Radiology 2011;259:720-9.

24. Wang Y, Li L, Wang YX, et al. Ultrasound findings of papillary thyroid microcarcinoma: a review of 113 consecutive cases with histopathologic correlation. Ultrasound Med Biol 2012;38:1681-8.

25. Park KN, Kang KY, Hong HS, et al. Predictive Value of Estimated Tumor Volume Measured by Ultrasonography for Occult Central Lymph Node Metastasis in Papillary Thyroid Carcinoma. Ultrasound Med Biol 2015;41:2849-54.

26. Som PM, Curtin HD, Mancuso AA. An imaging-based classification for the cervical nodes designed as an adjunct 
to recent clinically based nodal classifications. Arch Otolaryngol Head Neck Surg 1999;125:388-96.

27. Solorzano CC, Carneiro DM, Ramirez M, et al. Surgeonperformed ultrasound in the management of thyroid malignancy. Am Surg 2004;70:576-80; discussion 580-2.

28. Sakaguchi Y, Mizoguchi N, Mitsumoto T, et al. A simple table lookup method for PET/CT partial volume correction using a point-spread function in diagnosing lymph node metastasis. Ann Nucl Med 2010;24:585-91.

29. Law TT, Lang BH. Incidental thyroid carcinoma by FDGPET/CT: a study of clinicopathological characteristics. Ann Surg Oncol 2011;18:472-8.

30. Sachpekidis C, Dettmer MS, Weidner S, et al. 18F-FDG PET/CT of Papillary Carcinoma in a Lateral Thyroglossal Duct Cyst. Clin Nucl Med 2017;42:e371-4.

31. Eun NL, Son EJ, Kim JA, et al. Comparison of the diagnostic performances of ultrasonography, CT and fine needle aspiration cytology for the prediction of lymph node metastasis in patients with lymph node dissection of papillary thyroid carcinoma: A retrospective cohort study. Int J Surg 2018;51:145-50.

32. Suh CH, Baek JH, Choi YJ, et al. Performance of CT in the Preoperative Diagnosis of Cervical Lymph Node Metastasis in Patients with Papillary Thyroid Cancer: A Systematic Review and Meta-Analysis. AJNR Am J Neuroradiol 2017;38:154-61.

33. Ahn JE, Lee JH, Yi JS, et al. Diagnostic accuracy of CT and ultrasonography for evaluating metastatic cervical lymph nodes in patients with thyroid cancer. World J Surg
2008;32:1552-8.

34. Han ZJ, Shu YY, Lai XF, et al. Value of computed tomography in determining the nature of papillary thyroid microcarcinomas: evaluation of the computed tomographic characteristics. Clin Imaging 2013;37:664-8.

35. Park JE, Lee JH, Ryu KH, et al. Improved Diagnostic Accuracy Using Arterial Phase CT for Lateral Cervical Lymph Node Metastasis from Papillary Thyroid Cancer. AJNR Am J Neuroradiol 2017;38:782-8.

36. Scott GC, Meier DA, Dickinson CZ. Cervical lymph node metastasis of thyroid papillary carcinoma imaged with fluorine-18-FDG, technetium-99m-pertechnetate and iodine-131-sodium iodide. J Nucl Med 1995;36:1843-5.

37. Choi B, Kim DH, Son SH, et al. Usefulness of SPECT/ CT for equivocal findings on (131)I whole-body scan in a patient with differentiated papillary thyroid cancer. Clin Nucl Med 2014;39:e160-2.

38. Li L, Zhao Y, Luo D, et al. Diagnostic value of singlesource dual-energy spectral computed tomography in differentiating parotid gland tumors: initial results. Quant Imaging Med Surg 2018;8:588-96.

39. Miles KA. Tumour angiogenesis and its relation to contrast enhancement on computed tomography: a review. Eur J Radiol 1999;30:198-205.

40. Payabvash S. Quantitative diffusion magnetic resonance imaging in head and neck tumors. Quant Imaging Med Surg. 2018;8:1052-65.

41. Turner HE, Harris AL, Melmed S, et al. Angiogenesis in endocrine tumors. Endocr Rev 2003;24:600-32.
Cite this article as: Li L, Cheng SN, Zhao YF, Wang XY, Luo DH, Wang Y. Diagnostic accuracy of single-source dual-energy computed tomography and ultrasonography for detection of lateral cervical lymph node metastases of papillary thyroid carcinoma. J Thorac Dis 2019;11(12):5032-5041. doi: $10.21037 /$ jtd.2019.12.45 